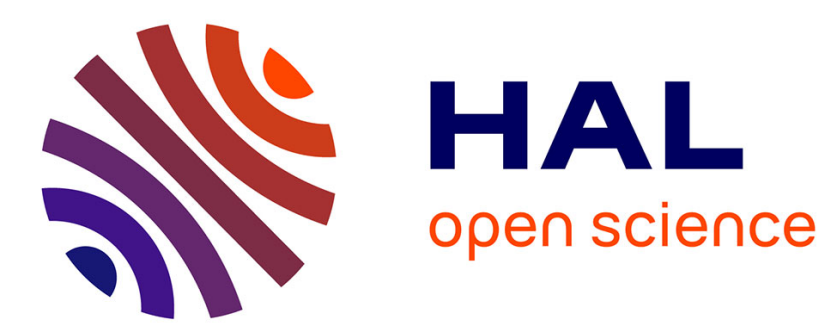

\title{
Sur la commande tolérante aux défauts des machines asynchrones: Une approche implicite
}

Omar Benzineb, Mohamed Tadjine, Mohamed Benbouzid, Demba Diallo

\section{To cite this version:}

Omar Benzineb, Mohamed Tadjine, Mohamed Benbouzid, Demba Diallo. Sur la commande tolérante aux défauts des machines asynchrones: Une approche implicite. European Journal of Electrical Engineering, 2012, 15 (6), pp.633-658. hal-00780768

\section{HAL Id: hal-00780768 https://hal.science/hal-00780768}

Submitted on 24 Jan 2013

HAL is a multi-disciplinary open access archive for the deposit and dissemination of scientific research documents, whether they are published or not. The documents may come from teaching and research institutions in France or abroad, or from public or private research centers.
L'archive ouverte pluridisciplinaire HAL, est destinée au dépôt et à la diffusion de documents scientifiques de niveau recherche, publiés ou non, émanant des établissements d'enseignement et de recherche français ou étrangers, des laboratoires publics ou privés. 


\title{
Sur la commande tolérante aux défauts des machines asynchrones: Une approche implicite
}

\author{
O. Benzineb* ${ }^{*}$ M. Tadjine** ${ }^{* *}$ M.E.H. Benbouzid ${ }^{*}-$ D. \\ Diallo**
}

*Université de Brest, EA 4325 LBMS

IUT de Brest - Rue de Kergoat - CS 93837, 29238 Brest Cedex 03

Omar.Benzineb@univ-brest.fr, Mohamed.Benbouzid@univ-brest.fr

${ }^{* *}$ Ecole Nationale Polytechnique d'Alger

Mohamed.Tadjine@mail.enp.edu.dz

${ }^{* * *}$ Laboratoire de Génie Electrique de Paris, CNRS UMR 8507, Supélec, Université Pierre et Marie Curie, Université Paris Sud

Demba.Diallo@1gep.supelec.fr

RÉSUMÉ. Dans cet article, deux approches de commande tolérante aux défauts (FTC FaultTolerant Control) sont étudiées et appliquées à la machine asynchrone. Dans ce contexte, la phase de détection et d'isolation du défaut est décalée par rapport à la phase de reconfiguration de la commande. Celle-ci est réalisée en testant l'état d'un modèle interne qui s'active automatiquement dès l'apparition d'un défaut pour compenser son effet. Cet effet peut être convenablement modélisé par un signal exogène issu d'un système autonome stable appelé exosystème. Une commande additive est ainsi ajoutée à la commande nominale. Issue du modèle interne, cette commande sert à compenser l'effet du défaut. La première approche FTC exploite un modèle interne basé sur l'équation de Sylvester qui entraîne une divergence lorsque la machine est affectée par deux défaut ou plus. La seconde approche, quant à elle, élimine le problème de divergence par un réglage adapté des matrices du système.

ABSTRACT. This paper deals with the application of implicit fault-tolerant control techniques to induction motor drives using a Backstepping approach. For that purpose, the induction motor, the disturbances as well as the faults signals have been modeled. A Backstepping control strategy (nominal control) is then synthesized and applied to the induction motor drive for robust control purposes. For fault-tolerant control purposes, an additive control term is generated from an internal state model in order to compensate for the fault effects. Simulations carried-out on a 1.1-kW induction motor drive clearly show the effectiveness of the proposed approaches.

MOTS-CLÉS : Machine asynchrone, modèle interne, défauts, commande tolérante aux défauts, commande par Backstepping.

KEYWORDS: Induction machine, internal model, fault, fault-tolerant control (FTC), Backstepping control. 


\section{Nomenclature}

$\begin{array}{lll}\alpha, \beta & = & \text { Indice du référentiel stationnaire } ; \\ d, q & = & \text { Indices du référentiel synchrone; } \\ s,(r) & = & \text { Indice stator (rotor); } \\ i & = & \text { Courant ; } \\ \Phi & = & \text { Flux ; } \\ \omega_{r} & = & \text { Vitesse électrique rotorique ; } \\ C_{r} & = & \text { Couple résistant ; } \\ R & = & \text { Résistance ; } \\ L(M) & = & \text { Inductance (inductance mutuelle); } \\ T & = & \text { Constante de temps }\left(T_{s}=L_{s} / R_{s}, T_{r}=L_{r} / R_{r}\right) ; \\ \sigma & = & \text { Coefficient de dispersion } \sigma=1-M^{2} / L_{s} L_{r} ; \\ f & = & \text { Frottements visqueux ; } \\ J & = & \text { Inertie rotorique ; } \\ p & = & \text { Nombre de paires de pôles. }\end{array}$

\section{Introduction}

L'augmentation de la fiabilité, la disponibilité et la sûreté de fonctionnement, constitue à l'heure actuelle, l'une des préoccupations majeures des industriels. Dans certains systèmes complexes, comme dans l'aéronautique ou les centrales nucléaires, la phase de détection et de localisation d'un ou de plusieurs défauts est nécessaire mais n'est pas suffisante pour garantir la sûreté de fonctionnement. En effet, il est indispensable de modifier la loi de commande pour assurer la sûreté de fonctionnement et maintenir le niveau minimal de performances. On parle alors de commande tolérante aux défauts (Zhang Y. et al., 2008 et Campos-Delgado D.U. et al., 2008).

Les machines électriques sont des éléments critiques dans les processus industriels mais également dans les applications liées au transport. Elles peuvent être le siège de défauts d'origine mécanique et électrique ayant des signatures fréquentielles liées aux paramètres structurels de la machine (Benbouzid M.E.H. et al., 2003 et Benbouzid M.E.H., 2000). Or ces paramètres changent au cours du temps du fait de la température, des conditions d'exploitation, etc. Il est donc indispensable de disposer d'une loi de commande nominale robuste vis-à-vis des variations paramétriques. Ainsi, le modèle interne sera essentiellement sensible aux défauts.

La structure de la commande tolérante associe donc un bloc de détection et de diagnostic (Fault Detection and Diagnosis - FDD) à une stratégie de reconfiguration (Fault-Tolerant Control - FTC) (Eva Wu N., 2000). Il existe dans la littérature deux approches, l'une dite explicite et l'autre implicite. L'approche explicite nécessite un bloc FDD performant dans lequel les modèles des défauts des constituants de la chaîne de conversion d'énergie sont connus et validés (Fekih, A., 2008 ; Benbouzid 
M.E.H. et $a l ., 2007$ et Jeong Y.S. et al.). Par contre dans l'approche implicite, on ne s'intéresse qu'aux impacts des défauts. C'est au sein de la fonction FTC que l'on met en œuvre un modèle interne tel que la loi de commande réagisse de façon adéquate pour annuler ou du moins atténuer l'effet du défaut sans pour autant avoir une idée précise ni de la localisation ni du type de défaut. On peut donc noter que dans le cas implicite on peut se passer à priori de la fonction FDD (Bonivento C. et al., 2004).

Nous avons donc dans cet article développé une commande par la technique du Backstepping robuste vis-à-vis des variations paramétriques et du couple de charge (Tadjine M. et al., 2007 ; Ebrahim A. et al., 2006 et Tan H. et al., 1999). Afin de concevoir le bloc FTC, nous l'avons associé à un modèle interne qui génère un terme additif pour compenser l'effet des défauts. Le niveau de la compensation est un indicateur de la sévérité du défaut et la nature de la compensation est une aide au diagnostic.

\section{Modélisation de la machine, des variations paramétriques et des défauts}

\subsection{Modélisation de la machine}

La modélisation de la machine asynchrone est faite sous forme de représentation d'état dans un référentiel stationnaire $\alpha-\beta$. Les variables d'états sont les courants statoriques, les flux rotoriques et la vitesse électrique du rotor $\omega_{r}$ et le vecteur d'entrées est constitué par les tensions statoriques. Le modèle de la machine asynchrone peut se mettre sous la forme d'état suivante (Krause P.C. et al., 2002).

$$
\left\{\begin{array}{l}
\dot{x}=f(x)+B u+D C_{r} \\
f=\left[\begin{array}{lllll}
f_{1} & f_{2} & f_{3} & f_{4} & f_{5}
\end{array}\right]^{T} \\
x=\left[\begin{array}{lllll}
x_{1} & x_{2} & x_{3} & x_{4} & x_{5}
\end{array}\right]^{T}=\left[\begin{array}{lllll}
i_{s \alpha} & \mathrm{i}_{\mathrm{s} \beta} & \Phi_{r \alpha} & \Phi_{r \beta} & \omega_{r}
\end{array}\right]^{T} \\
u=\left[\begin{array}{lll}
u_{1} & u_{2}
\end{array}\right]^{T} \\
B=\left[\begin{array}{lllll}
b_{1} & 0 & 0 & 0 & 0 \\
0 & b_{2} & 0 & 0 & 0
\end{array}\right]^{T} \\
D=\left[\begin{array}{lllll}
0 & 0 & 0 & 0 & d_{1}
\end{array}\right]^{T}
\end{array}\right.
$$

Avec le champ de vecteur $f(x)$ donné par : 


$$
\left\{\begin{array}{l}
f_{1}(x)=a_{1} x_{1}+a_{2} x_{3}+a_{3} x_{4} x_{5} \\
f_{2}(x)=a_{4} x_{2}+a_{5} x_{3} x_{5}+a_{6} x_{4} \\
f_{3}(x)=a_{7} x_{1}+a_{8} x_{3}+a_{9} x_{4} x_{5} \\
f_{4}(x)=a_{10} x_{2}+a_{11} x_{3} x_{5}+a_{12} x_{4} \\
f_{5}(x)=a_{13} x_{1} x_{4}+a_{14} x_{2} x_{3}+a_{15} x_{5}
\end{array}\right.
$$

Les composantes de ce vecteur s'expriment en fonction des paramètres de la machine asynchrone comme suit :

$$
\left\{\begin{array}{l}
a_{1}=a_{4}=-\left(\frac{1}{T_{s} \sigma}+\frac{1-\sigma}{T_{r} \sigma}\right), a_{2}=a_{6}=\frac{1-\sigma}{T_{r} M \sigma}, a_{3}=-a_{5}=\frac{1-\sigma}{M \sigma} \\
a_{7}=a_{10}=\frac{M}{T_{r}}, a_{8}=a_{12}=-\frac{1}{T_{r}}, a_{9}=-a_{11}=-1 \\
a_{13}=-a_{14}=-p^{2} \frac{M}{J L_{r}}, a_{15}=-\frac{f}{J} \\
b_{1}=b_{2}=\frac{1}{\sigma L_{s}}, d_{1}=-p \frac{1}{J}
\end{array}\right.
$$

\subsection{Modélisation des variations paramétriques}

Un terme $\Delta\left(x, \Delta a_{i}\right)$ inconnu (lié à l'impact des variations paramétriques sur l'état) mais tout le temps borné s'ajoute dans le modèle [1] qui prend alors la forme :

$$
\dot{x}=f(x)+B u+D C_{r}+\Delta\left(x, \Delta a, \Delta b, \Delta d_{1}\right)
$$

Une façon de simuler l'effet des variations paramétriques sur le comportement de la machine est de provoquer à un instant donné, un changement aléatoire dans les coefficients du système d'équations [2]. Ce qui engendre les variations suivantes :

$$
\left\{\begin{array}{l}
a_{i} \rightarrow a_{i}^{0}+\Delta a_{i} ; i: 1, . .5 \\
b_{i} \rightarrow b_{i}^{0}+\Delta b_{i} ; i: 1, . .5 \\
d_{1} \rightarrow d_{1}^{0}+\Delta d_{1}
\end{array}\right.
$$

Les perturbations $\Delta\left(x, \Delta a, \Delta b, \Delta d_{1}\right)$ prendront la forme suivante : 


$$
\Delta\left(x, \Delta a, \Delta b, \Delta d_{1}\right)=\left[\begin{array}{c}
\Delta a_{1} x_{1}+\Delta a_{2} x_{3}+\Delta a_{3} x_{4} x_{5}+\Delta b_{1} u_{1} \\
\Delta a_{4} x_{2}+\Delta a_{5} x_{3} x_{5}+\Delta a_{6} x_{4}+\Delta b_{2} u_{2} \\
\Delta a_{7} x_{1}+\Delta a_{8} x_{3}+\Delta a_{9} x_{4} x_{5} \\
\Delta a_{10} x_{2}+\Delta a_{11} x_{3} x_{5}+\Delta a_{12} x_{4} \\
\Delta a_{13} x_{1} x_{4}+\Delta a_{14} x_{2} x_{3}+\Delta a_{15} x_{5}+\Delta d_{1} C_{r}
\end{array}\right]
$$

L'application de la différentielle totale exacte nous donne les variations des composantes $a_{i}, b_{i}$ et $d_{1}$.

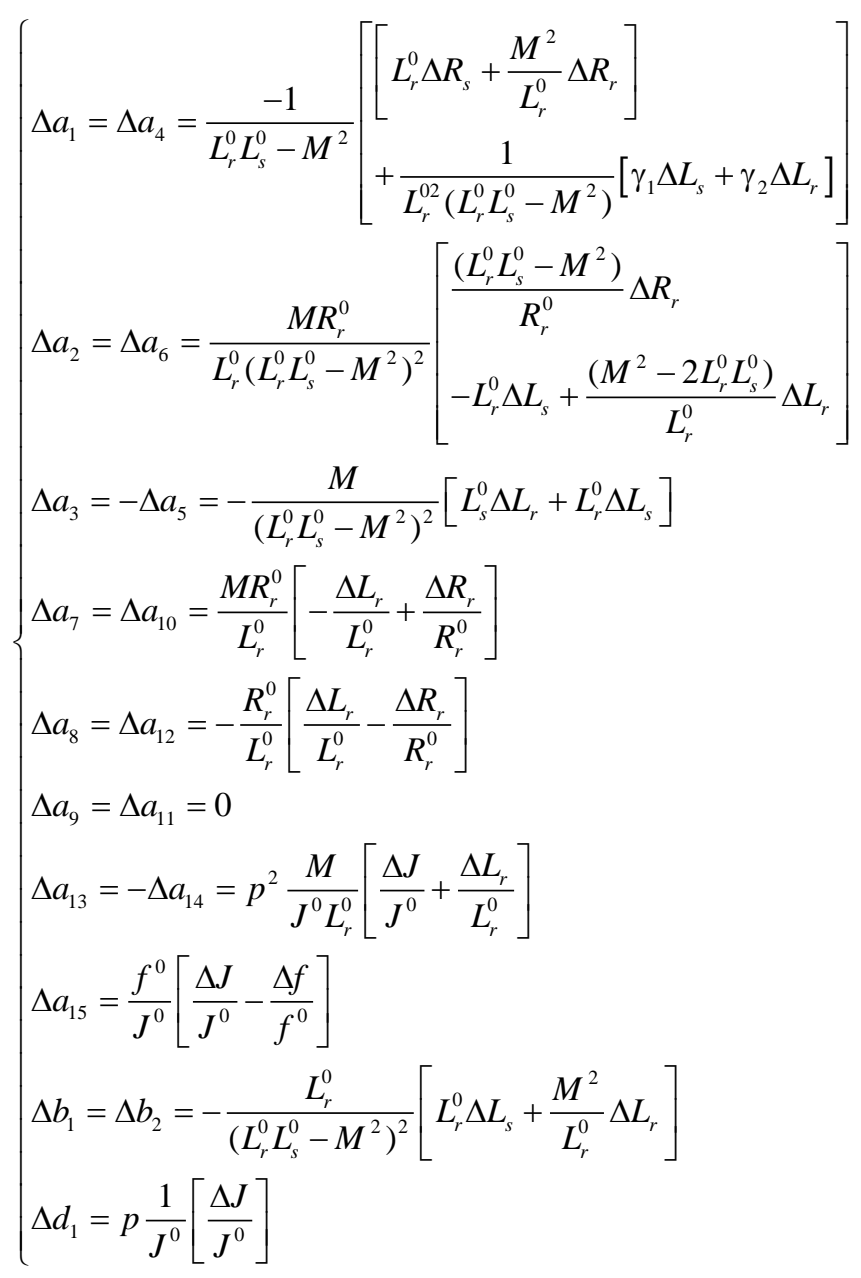

Où $X^{0}$ désigne les valeurs initiales des paramètres et 


$$
\left\{\begin{array}{l}
\gamma_{1}=L_{r}^{02}\left[R_{s}^{0}\left(L_{r}^{0}\right)^{2}+R_{r}^{0} M^{2}\right] \\
\gamma_{2}=M^{2}\left[-R_{s}\left(L_{r}^{0}\right)^{22}+\left(M^{2}-2 L_{r}^{0} L_{s}^{0}\right) R_{r}^{0}\right]
\end{array}\right.
$$

Les variations des inductances $L_{s}$ et $L_{r}$ intègrent celles de la mutuelle $M$.

\subsection{Modélisation des défauts}

Un terme $V$ représentant une perturbation inconnue mais tout le temps borné qui résulte de la présence d'un ou de plusieurs défauts dans la machine - s'ajoute au modèle [1] qui s'écrira alors - en absence de perturbations paramétriques - sous la forme :

$$
\dot{x}=f(x)+B u+D C_{r}+\eta V
$$

En absence de défauts, $V$ est identiquement nul et on a :

$$
\left\{\begin{array}{l}
\eta=\left[\begin{array}{lllll}
1 & 0 & 0 & 0 & 0 \\
0 & 1 & 0 & 0 & 0
\end{array}\right]^{T} \\
V=\left[\begin{array}{l}
V_{1} \\
V_{2}
\end{array}\right]
\end{array}\right.
$$

sous l'hypothèse que les effets des défauts se manifestent principalement sur les courants qui sont de plus les grandeurs les plus couramment accessibles par la mesure.

Les défauts envisagés par la suite se limitent à ceux dont la signature est fréquentielle. Dans ce cas, une façon simple d'introduire dans l'équation d'état du système [1] les harmoniques de défauts qui affectent les courants statoriques est d'utiliser un système différentiel linéaire stable représenté sous forme d'état :

$$
\dot{z}=S z
$$

La matrice dynamique $S$ est la seule caractéristique connue du système. Elle est constituée des pulsations $\omega_{i}=2 \pi f_{i}$ où $f_{i}$ présente la fréquence caractéristique du défaut.

$$
\left\{\begin{array}{l}
S=\operatorname{diag}\left(S_{i}\right) \\
S_{i}=\left[\begin{array}{cc}
0 & \omega_{i} \\
-\omega_{i} & 0
\end{array}\right] \\
i=1, \ldots, n_{f}
\end{array}\right.
$$


On peut alors écrire le vecteur $V$ des perturbations dues aux défauts sous la forme matricielle suivante :

$$
V=-\Gamma z=\left[\begin{array}{c}
a_{1} Q_{d}-Q_{d} S \\
a_{4} Q_{q}-Q_{q} S
\end{array}\right] z
$$

dans laquelle les matrices $Q_{d}$ et $Q_{q}$ de dimensions $\left(1 \times 2 n_{f}\right)$ valent respectivement :

$$
\left\{\begin{array}{l}
Q_{d}=\left[\begin{array}{llllll}
1 & 0 & 1 & 0 \ldots . & 0 & 1
\end{array}\right] \\
Q_{q}=\left[\begin{array}{llllll}
0 & 1 & 0 & 1 \ldots . & 1 & 0
\end{array}\right]
\end{array}\right.
$$

Finalement en présence de défauts et de perturbations paramétriques, le modèle de la machine asynchrone devient :

$$
\dot{x}=f(x)+B u+D C_{r}+\Delta\left(x, \Delta a, \Delta b, \Delta d_{1}\right)+\eta V
$$

C'est ce modèle qui sera par la suite utilisé dans les simulations numériques. Dans la partie suivante, nous allons développer la loi de commande de la machine asynchrone.

\section{Commande par Backstepping}

L'approche Backstepping, appliquée à la commande de la machine asynchrone, est basée sur le principe de l'orientation du flux rotorique (Tadjine M. et al., 2007 ; Ebrahim A. et al., 2006 et Tan H. et al., 1999).

\subsection{Principe de la commande à flux orienté}

L'objectif de la commande à flux orienté est d'avoir un couple électromagnétique proportionnel à la composante en quadrature du courant de la machine. Cette méthode consiste à orienter le flux rotorique suivant la direction de l'axe $\alpha$ (Leonhard W., 1996).

La position du rotor est définie par l'angle $\theta_{s}$ comme suit :

$$
\theta_{s}=\arctan \left(\frac{\Phi_{r \beta}}{\Phi_{r \alpha}}\right)
$$

La transformation du repère $\alpha-\beta$ vers le repère $d-q$ se fait comme suit : 


$$
\left[\begin{array}{c}
x_{s d} \\
x_{s q}
\end{array}\right]=\left[\begin{array}{cc}
\cos \theta_{s} & \sin \theta_{s} \\
-\sin \theta_{s} & \cos \theta_{s}
\end{array}\right]\left[\begin{array}{c}
x_{s \alpha} \\
x_{s \beta}
\end{array}\right]
$$

$x$ pouvant être le courant, la tension ou le flux. A partir de [11], on trouve :

$$
\overline{\Phi_{r}}=\Phi_{r \alpha}+j \Phi_{r \beta}=\sqrt{\Phi_{r \alpha}^{2}+\Phi_{r \beta}^{2}}\left[\cos \theta_{s}+j \sin \theta_{s}\right]
$$

L'équation [17] pourrait alors s'écrire pour les courants, les tensions et les flux sous la forme suivante :

$$
\left\{\begin{array}{l}
i_{s d}=\frac{\Phi_{r \alpha} i_{s \alpha}-\Phi_{r \beta} i_{s \beta}}{\sqrt{\Phi_{r \alpha}^{2}+\Phi_{r \beta}^{2}}}, i_{s q}=\frac{\Phi_{r \alpha} i_{s \beta}-\Phi_{r \beta} i_{s \alpha}}{\sqrt{\Phi_{r \alpha}^{2}+\Phi_{r \beta}^{2}}} \\
\Phi_{r d}=\sqrt{\Phi_{r \alpha}^{2}+\Phi_{r \beta}^{2}}, \Phi_{r q}=0 \\
V_{s d}=u_{1}=\frac{V_{s \alpha} \Phi_{r \alpha}-V_{s \beta} \Phi_{r \beta}}{\sqrt{\Phi_{r \alpha}^{2}+\Phi_{r \beta}^{2}}}, V_{s q}=u_{2}=\frac{V_{s \beta} \Phi_{r \alpha}-V_{s \alpha} \Phi_{r \beta}}{\sqrt{\Phi_{r \alpha}^{2}+\Phi_{r \beta}^{2}}}
\end{array}\right.
$$

Le nouveau modèle de la machine dans le repère $d$ - $q$ est donné par :

$$
\begin{aligned}
& \left\{\begin{array}{l}
\dot{x}_{1}=a_{1} x_{1}+\dot{\theta}_{s} x_{2}+a_{2} x_{3}+b u_{1} \\
\dot{x}_{2}=-\dot{\theta}_{s} x_{1}+a_{1} x_{2}+a_{5} x_{3} x_{5}+b u_{2} \\
\dot{x}_{3}=a_{8} x_{3}+a_{10} x_{1} \\
\dot{x}_{4}=0 \\
\dot{x}_{5}=a_{14} x_{2} x_{3}+a_{15} x_{5}+d_{1} C_{r}
\end{array}\right.
\end{aligned}
$$

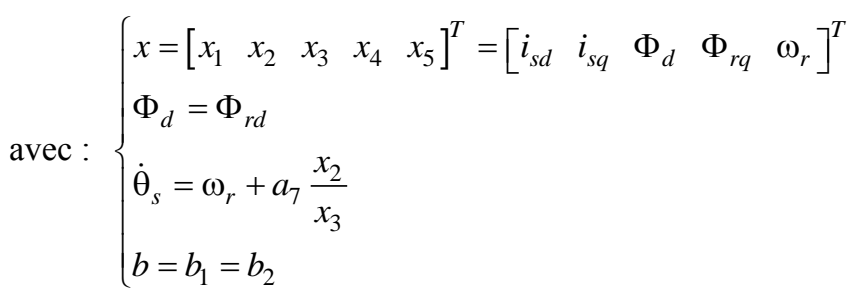

\subsection{Commande par Backstepping}

La technique de commande par Backstepping est une méthode de synthèse en non linéaire. Il s'agit de choisir au départ une fonction de Lyapunov pour le premier 
sous-système et de l'augmenter au fur et à mesure que l'on stabilise les différents sous-systèmes successifs, pour aboutir enfin à une fonction de Lyapunov globale qui stabilise le système global. L'application du Backstepping à la commande de la machine asynchrone est divisée en trois étapes.

\section{Etape 1}

Cette première étape consiste à identifier les erreurs $\varepsilon_{1}$ (écart entre la référence $\omega_{\text {ref }}$ et la vitesse électrique réelle $\left.\omega_{r}\right)$ et $\varepsilon_{2}$ (écart entre la référence $\left(\Phi_{d}\right)_{r e f}$ et le module du flux $\Phi_{d}$ ).

$$
\left\{\begin{array}{l}
\varepsilon_{5}=\left(x_{5}\right)_{r e f}-x_{5} \\
\varepsilon_{3}=\left(x_{3}\right)_{r e f}-x_{3}
\end{array}\right.
$$

Les dérivées des erreurs sont données par :

$$
\left\{\begin{array}{l}
\dot{\varepsilon}_{5}=\left(\dot{x}_{5}\right)_{r e f}-a_{14} \cdot x_{2} x_{3}-a_{15} x_{5}-d_{1} C_{r} \\
\dot{\varepsilon}_{3}=\left(\dot{x}_{3}\right)_{r e f}-a_{8} x_{3}-a_{10} x_{1}
\end{array}\right.
$$

La première fonction de Lyapunov est définie par :

$$
v_{1}=\frac{1}{2}\left(\varepsilon_{5}^{2}+\varepsilon_{3}^{2}\right)
$$

En supposant que le couple de charge est connu ou estimé, on choisit les fonctions stabilisantes comme suit :

$$
\left\{\begin{array}{l}
\left(x_{2}\right)_{r e f}=\frac{1}{a_{14} x_{3}}\left(k_{5} \varepsilon_{5}+\dot{x}_{5}^{d}-a_{15} x_{5}-d_{1} C_{r}\right) \\
\left(x_{1}\right)_{r e f}=\frac{1}{a_{10}}\left(k_{3} \varepsilon_{3}+\dot{x}_{3}^{d}-a_{8} x_{3}\right)
\end{array}\right.
$$

Alors, si les courants suivent parfaitement leurs références, la dynamique des erreurs sera donnée par :

$$
\left\{\begin{array}{l}
\dot{\varepsilon}_{5}=-k_{5} \varepsilon_{5} \\
\dot{\varepsilon}_{3}=-k_{3} \varepsilon_{3}
\end{array}\right.
$$

Ainsi donc, la dérivée de la fonction de Lyapunov par rapport au temps donnée par : 


$$
\dot{v}_{1}=-k_{5} \varepsilon_{5}^{2}-k_{3} \varepsilon_{3}^{2}<0
$$

est négative pour $\left\{\begin{array}{l}k_{5}>0 \\ k_{3}>0\end{array}\right.$

\section{Etape 2}

Dans cette étape, à partir de [25] on définit deux nouvelles erreurs des composantes du courant statorique données par :

$$
\left\{\begin{array}{l}
\varepsilon_{1}=\left(x_{1}\right)_{r e f}-x_{1}=\frac{1}{a_{10}}\left(k_{3} \varepsilon_{3}+\dot{x}_{3}^{d}-a_{8} x_{3}\right)-x_{1} \\
\varepsilon_{2}=\left(x_{2}\right)_{r e f}-x_{2}=\frac{1}{a_{14} x_{3}}\left(k_{5} \varepsilon_{5}+\dot{x}_{5}^{d}-a_{15} x_{5}-d_{1} C_{r}\right)-x_{2}
\end{array}\right.
$$

Alors [23] prendra la forme :

$$
\left\{\begin{array}{l}
\dot{\varepsilon}_{5}=-k_{5} \varepsilon_{5}+a_{14} x_{3} \varepsilon_{3} \\
\dot{\varepsilon}_{3}=-k_{3} \varepsilon_{3}+a_{10} \varepsilon_{1}
\end{array}\right.
$$

La dérivée de [28] nous donne :

$$
\begin{gathered}
\left\{\begin{array}{l}
\dot{\varepsilon}_{1}=\left(\dot{x}_{1}\right)_{r e f}-h_{1}(x)-b u_{1} \\
\dot{\varepsilon}_{2}=\left(\dot{x}_{2}\right)_{r e f}-h_{2}(x)-b u_{2}
\end{array}\right. \\
\text { où }\left\{\begin{array}{l}
h_{1}(x)=a_{1} x_{1}+\omega_{a} x_{2}+a_{2} x_{3} \\
h_{2}(x)=-\omega_{a} x_{1}+a_{1} x_{2}+a_{5} x_{3} x_{5}
\end{array}\right.
\end{gathered}
$$

\section{Etape 3}

Pour définir les lois de commande, on adopte une nouvelle fonction de Lyapunov décrite par l'expression suivante :

$$
v_{2}=\frac{1}{2}\left(\varepsilon_{1}^{2}+\varepsilon_{2}^{2}+\varepsilon_{3}^{2}+\varepsilon_{4}^{2}\right)
$$

Ainsi la dérivée de la fonction de Lyapunov finale s'exprimera comme :

$$
\dot{v}_{2}=\varepsilon_{1} \dot{\varepsilon}_{1}+\varepsilon_{2} \dot{\varepsilon}_{2}+\varepsilon_{3} \dot{\varepsilon}_{3}+\varepsilon_{4} \dot{\varepsilon}_{4}
$$


et sera donnée par :

$$
\left\{\begin{aligned}
& \dot{v}_{2}=-k_{1} \varepsilon_{1}^{2}-k_{2} \varepsilon_{2}^{2}-k_{3} \varepsilon_{3}^{2}-k_{4} \varepsilon_{4}^{2} \\
&+\varepsilon_{2}\left(k_{2} \varepsilon_{2}+\left(\dot{x}_{2}\right)_{r e f}-h_{2}(x)-b u_{2}+a_{14} x_{3} \varepsilon_{5}\right) \\
&+\varepsilon_{1}\left(k_{1} \varepsilon_{1}+\left(\dot{x}_{1}\right)_{r e f}-h_{1}(x)-b u_{1}+a_{10} \varepsilon_{3}\right) \\
& k_{2}>0 \\
& k_{1}>0
\end{aligned}\right.
$$

On choisit la commande comme suit :

$$
\left\{\begin{array}{l}
u_{1}=V_{s d}=\frac{1}{b}\left[\left(\dot{x}_{1}\right)_{r e f}+k_{1} \varepsilon_{1}-h_{1}(x)+a_{10} \varepsilon_{3}\right] \\
u_{2}=V_{s q}=\frac{1}{b}\left[\left(\dot{x}_{2}\right)_{r e f}+k_{2} \varepsilon_{2}-h_{2}(x)+a_{14} x_{3} \varepsilon_{5}\right]
\end{array}\right.
$$

Les dérivées des erreurs $\varepsilon_{2}$ et $\varepsilon_{1}$ deviennent :

$$
\left\{\begin{array}{l}
\dot{\varepsilon}_{1}=-a_{10} \varepsilon_{3}-k_{1} \varepsilon_{1} \\
\dot{\varepsilon}_{2}=-a_{14} x_{3} \varepsilon_{5}-k_{2} \varepsilon_{2}
\end{array}\right.
$$

Donc à partir de [29] et [36] et en introduisant l'erreur fictive $\varepsilon_{4}$ on peut écrire :

$$
\dot{\varepsilon}=\left[\begin{array}{c}
\dot{\varepsilon}_{1} \\
\dot{\varepsilon}_{2} \\
\dot{\varepsilon}_{3} \\
\dot{\varepsilon}_{4} \\
\dot{\varepsilon}_{5}
\end{array}\right]=\left[\begin{array}{ccccc}
-k_{1} & 0 & -a_{10} & 0 & 0 \\
0 & -k_{2} & 0 & 0 & -a_{14} x_{3} \\
a_{10} & 0 & -k_{3} & 0 & 0 \\
0 & 0 & 0 & 0 & 0 \\
0 & a_{14} x_{3} & 0 & 0 & -k_{5}
\end{array}\right]\left[\begin{array}{c}
\varepsilon_{1} \\
\varepsilon_{2} \\
\varepsilon_{3} \\
\varepsilon_{4} \\
\varepsilon_{5}
\end{array}\right]=A \varepsilon
$$

La Figure 1 illustre le principe de la commande par Backstepping de la machine asynchrone. La première étape de la commande consiste à générer les courants de référence $\left(i_{s d}\right)_{r e f}$ et $\left(i_{s q}\right)_{r e f}$, représentant la commande fictive. On adapte ensuite la loi de commande $V_{s d}$ et $V_{s q}$ à partir de [35] pour assurer la stabilité de la machine. Les flux $\Phi_{r \alpha}$ et $\Phi_{r \beta}$ sont déterminés à partir des relations suivantes :

$$
\left\{\begin{array}{l}
\Phi_{r \alpha}=\frac{L_{r}}{M}\left[\left(\int\left(V_{s \alpha}-R_{s} i_{s \alpha}\right) d t\right)-\sigma L_{s} i_{s \alpha}\right] \\
\Phi_{r \beta}=\frac{L_{r}}{M}\left[\left(\int\left(V_{s \beta}-R_{s} i_{s \beta}\right) d t\right)-\sigma L_{s} i_{s \beta}\right]
\end{array}\right.
$$




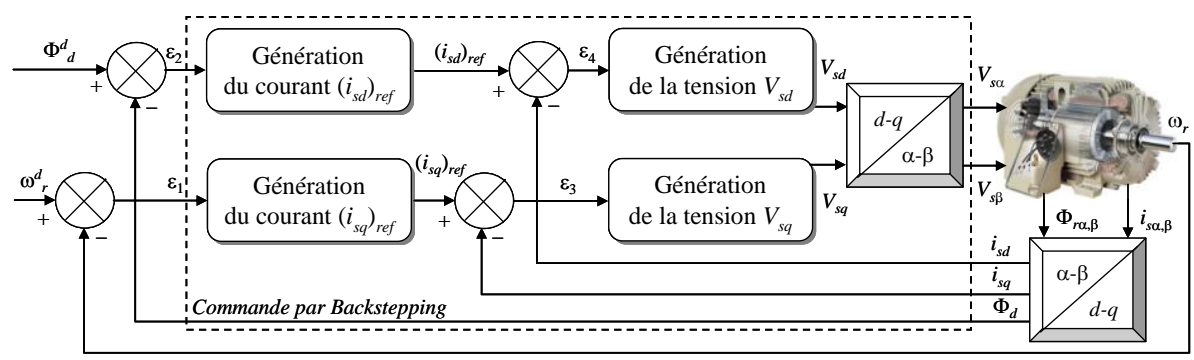

Figure 1. Principe de la commande par Backstepping de la machine asynchrone.

\section{Commande implicitement tolérante aux défauts}

Un système tolérant aux défauts est un système capable de détecter la présence de défauts et de s'adapter automatiquement en étant capable de maintenir la stabilité et au mieux les performances nominales du système (Muenchhof M. et al., 2009).

La Figure 2 représente le principe d'une commande implicitement tolérante aux défauts. Elle suppose que les effets des défauts sur le système peuvent être convenablement modélisés par un signal exogène issu d'un système autonome stable appelé exosystème. Une commande additive, ajoutée à la commande nominale, sert à compenser l'effet du défaut (aspect FTC). Cette commande additive est issue d'un modèle interne dont le rôle est de reproduire le signal représentant l'effet du défaut.

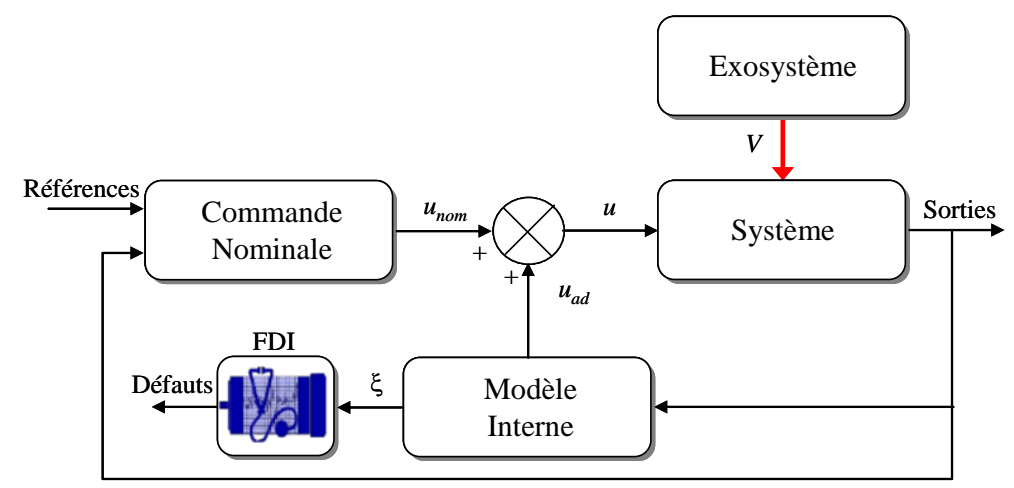

Figure 2. Structure d'une commande implicitement tolérante aux défauts.

L'équation d'état du système est la suivante :

$$
\dot{x}=f(x)+B u_{d q}+\eta V+\Delta\left(x, \Delta a, \Delta b, \Delta d_{1}\right)+D C_{r}
$$

Si le couple de charge et les perturbations paramétriques sont compensés par la commande nominale déterminée par [35] alors [39] devient : 


$$
\dot{x}=f(x)+B u_{d q}+\eta V
$$

et l'objectif est alors de calculer un terme $u_{a d}$ à rajouter à la commande nominale pour compenser l'effet des défauts. Ce terme est nul en l'absence de défauts. La nouvelle commande s'exprime alors par :

$$
u=\left[\begin{array}{l}
u_{1} \\
u_{2}
\end{array}\right]=\left[\begin{array}{l}
u_{1 \text { nom }} \\
u_{2 \text { nom }}
\end{array}\right]+\left[\begin{array}{l}
u_{1 a d} \\
u_{2 a d}
\end{array}\right]+\left[\begin{array}{l}
u_{1 c} \\
u_{2 c}
\end{array}\right]
$$

En choisissant le terme $u_{c}$ tel que

$$
u_{c}=\left[\begin{array}{l}
u_{1 c} \\
u_{2 c}
\end{array}\right]=\left[\begin{array}{c}
a_{10} \tilde{x}_{3} \\
a_{14} x_{3} \tilde{x}_{5}
\end{array}\right] \text {, }
$$

il permet de donner une forme adéquate à l'erreur pour calculer la commande additionnelle $u_{a d}$.

La différence instantanée entre la dérivée de l'état du système et de la consigne devient :

$$
\dot{\tilde{x}}=\left[\begin{array}{c}
\dot{\tilde{x}}_{1} \\
\dot{\tilde{x}}_{2} \\
\dot{\tilde{x}}_{3} \\
\dot{\tilde{x}}_{4} \\
\dot{\tilde{x}}_{5}
\end{array}\right]=\left[\begin{array}{c}
\left(\dot{x}_{1}\right)_{\text {ref }} \\
\left(\dot{x}_{2}\right)_{\text {ref }} \\
\left(\dot{x}_{3}\right)_{\text {ref }} \\
\left(\dot{x}_{4}\right)_{\text {ref }} \\
\left(\dot{x}_{5}\right)_{\text {ref }}
\end{array}\right]-\left[\begin{array}{l}
\dot{x}_{1} \\
\dot{x}_{2} \\
\dot{x}_{3} \\
\dot{x}_{4} \\
\dot{x}_{5}
\end{array}\right]=\left\{\begin{array}{l}
-k_{1} \tilde{x}_{1}+b_{1} u_{1 a d}-\Gamma_{1} z_{1} \\
-k_{2} \tilde{x}_{2}+b_{2} u_{2 a d}-\Gamma_{2} z_{2} \\
-k_{3} \tilde{x}_{3}+a_{10} \tilde{x}_{1} \\
0 \\
-k_{5} \tilde{x}_{5}+a_{14} \tilde{x}_{2} x_{3}
\end{array}\right.
$$

Remarquons que:

- dans la troisième équation si $\tilde{x}_{1} \rightarrow 0 \Rightarrow \tilde{x}_{3} \rightarrow 0$

- dans la quatrième équation on à $x_{4}=0 \Rightarrow\left(\dot{x}_{4}\right)_{r e f}=0$

- dans la cinquième équation si $\tilde{x}_{2} \rightarrow 0 \Rightarrow \tilde{x}_{5} \rightarrow 0$

Dans la suite, pour la détermination de $u_{a d}$ nous allons considérer le sous système suivant :

$$
\tilde{x}=\left[\begin{array}{l}
\tilde{x}_{1} \\
\tilde{x}_{2}
\end{array}\right]
$$

dont la dynamique se déduit de [43]. 


$$
\left\{\begin{array}{l}
\dot{z}=S z \\
\dot{\tilde{x}}=\left[\begin{array}{c}
\dot{\tilde{x}}_{1} \\
\dot{\tilde{x}}_{2}
\end{array}\right]=\left[\begin{array}{c}
-k_{1} \tilde{x}_{1}+b_{1} u_{1 a d}-\Gamma_{1} z_{1} \\
-k_{2} \tilde{x}_{2}+b_{2} u_{2 a d}-\Gamma_{2} z_{2}
\end{array}\right]
\end{array}\right.
$$

et qu'on peut l'écrire sous une forme matricielle :

$$
\dot{\tilde{x}}=H(\tilde{x})+\tilde{B} u_{a d}-\Gamma z
$$

$\operatorname{avec}\left\{\begin{array}{l}H(\tilde{x})=\tilde{A} \tilde{x}, \tilde{A}=\left[\begin{array}{cc}-k_{1} & 0 \\ 0 & -k_{2}\end{array}\right] \\ \tilde{B}=\left[\begin{array}{ll}b_{1} & 0 \\ 0 & b_{2}\end{array}\right]\end{array}\right.$

\subsection{Modèle interne}

En supposant que les pulsations caractéristiques des défauts $\omega_{i}\left(\right.$ de nombre $\left.n_{f}\right)$ et donc les matrices $S$ et $\Gamma$ soient parfaitement connues, considérons l'équation de Sylvester suivante (O’Leary D.P., 2005) :

$$
M S-F M=G \Gamma
$$

$F$ et $G$ sont des matrices de dimensions adéquates telles que $F$ est arbitraire stable (Hurwitz) et $G$ choisie telle que la paire $(F, G)$ soit commandable :

$$
\left\{\begin{array}{l}
\operatorname{dim}(F)=2 n_{f} \times 2 n_{f} \\
\operatorname{dim}(G)=2 n_{f} \times 2
\end{array}\right.
$$

$M$ est l'unique solution de l'équation de Sylvester et elle est non singulière. Le modèle interne prend alors la forme suivante ( $S$ connue) :

$$
\left\{\begin{array}{l}
\dot{\xi}=S \xi+N(\tilde{x}) \\
\operatorname{dim}(\xi)=\operatorname{dim}(z)=2 n_{f}
\end{array}\right.
$$

Pour le calcul de la commande additive nous introduisons une nouvelle variable.

$$
\left\{\begin{array}{l}
\chi=M .(\xi-z)-G \tilde{x} \\
\dot{\chi}=M .(\dot{\xi}-\dot{z})-G \dot{\tilde{x}}
\end{array}\right.
$$


Par substitution, on obtient :

$$
\dot{\chi}=M S .(\xi-z)+M N(\tilde{x})-G H(\tilde{x})-G \tilde{B} u_{a d}+G \Gamma z
$$

On choisit alors $u_{a d}$ de la forme :

$$
u_{a d}=\tilde{B}^{-1} \Gamma \xi
$$

et l'expression de $\dot{\chi}$ devient :

$$
\dot{\chi}=[M S-G \Gamma](\xi-z)+M N(\tilde{x})-G H(\tilde{x})
$$

En utilisant [48], on obtient l'équation suivante:

$$
\dot{\chi}=F M .(\xi-z)+M N(\tilde{x})-G H(\tilde{x})
$$

qui prend la forme suivante en introduisant le terme $\chi$ :

$$
\dot{\chi}=F \chi+F G \tilde{x}+M N(\tilde{x})-G H(\dot{\tilde{x}})
$$

Finalement en choisissant :

$$
N(\tilde{x})=M^{-1}[G H(x)-F G \tilde{x}]
$$

on obtient

$$
\dot{\chi}=F \chi
$$

Cette dernière équation assure que l'erreur globale $\chi$ tend asymptotiquement vers zéro car $F$ est une matrice de Hurwitz par construction.

En tenant compte de l'expression de $u_{a d}$ et de [46], on peut écrire :

$$
\dot{\tilde{x}}=\tilde{A} \tilde{x}+\Gamma(\xi-z)
$$

En utilisant [51], on obtient :

$$
\dot{\tilde{x}}=\left(\tilde{A}+\Gamma M^{-1} G\right) \tilde{x}+\Gamma M^{-1} \chi
$$

La variable $\chi$ tend vers zéro lorsque le temps $t$ tend vers l'infini d'après [58] et la matrice $\tilde{A}$ est stable par construction. Les gains $k_{1}$ et $k_{2}$ sont choisis de manière à 
avoir la matrice dynamique $\left(\tilde{A}+\Gamma M^{-1} G\right)$ toujours stable, ce qui assure la convergence de $\tilde{x}$ vers zéro. Nous aurons donc d'après [43]:

$$
\begin{aligned}
{[\tilde{x} \rightarrow 0} & \left.\Leftrightarrow\left(\tilde{x}_{1} \rightarrow 0 \wedge \tilde{x}_{2} \rightarrow 0\right)\right] \\
& \Rightarrow\left(\tilde{x}_{3} \rightarrow 0 \wedge \tilde{x}_{4} \rightarrow 0 \wedge \tilde{x}_{5} \rightarrow 0\right)
\end{aligned}
$$

Toutes les erreurs convergent alors asymptotiquement vers 0 . Les effets des défauts sont compensés par la commande additionnelle. Le système est dit alors tolérant aux défauts.

D'autre part, de [58] et [60], il découle directement que :

$$
(\xi-z)=M^{-1}[\chi+G \tilde{x}] \rightarrow 0
$$

Autrement dit, l'état du modèle interne reproduit exactement l'état inconnu a priori du système exogène simulant les défauts permettant ainsi le diagnostic a posteriori. C'est l'aspect Fault Detection and Isolation (FDI) de cette commande.

\subsection{Développement d'une nouvelle approche}

Nous proposons maintenant une nouvelle approche pour déterminer la commande additive. On suppose que les pulsations sont toujours parfaitement connues et on garde la même forme du modèle interne [50].

On considère [46] et on garde la même forme de la commande additive [53]. Dans ce cas, on a :

$$
\dot{\tilde{x}}=\tilde{A} \tilde{x}+\Gamma(\xi-z)
$$

On considère la nouvelle variable erreur d'observation des défauts:

$$
e=(\xi-z)
$$

Les équations décrivant la dynamique des erreurs en boucle fermée sont donc :

$$
\left\{\begin{array}{l}
\dot{\tilde{x}}=\tilde{A} \tilde{x}+\Gamma e \\
\dot{e}=S e+N(\tilde{x})
\end{array}\right.
$$

L'objectif est de trouver l'expression de $N(\tilde{x})$ qui annule l'erreur d'observation des défauts et permet en même temps de rejeter leur effet car elle annule aussi $\tilde{x}$. Soit la fonction de Lyapunov suivante : 


$$
V=\frac{1}{2} \tilde{x}^{T} \tilde{x}+\frac{1}{2} e^{T} e
$$

Après développement, $\dot{V}$ devient :

$$
\dot{V}=\tilde{x}^{T} \tilde{A} \tilde{x}+e^{T} \Gamma^{T} \tilde{x}+e^{T} N(\tilde{x})
$$

Dans ce cas le choix de $N(\tilde{x})$ est donnée par :

$$
N(\tilde{x})=-\Gamma^{T} \tilde{x}
$$

Enfin $\dot{V}$ s'écrit :

$$
\dot{V}=\tilde{x}^{T} \tilde{A} \tilde{x} \leq 0
$$

Le système [65] devient alors :

$$
\left\{\begin{array}{l}
\dot{\tilde{x}}=\tilde{A} \tilde{x}+\Gamma e \\
\dot{e}=-\Gamma^{t} \tilde{x}+S e
\end{array}\right.
$$

Et l'objectif de la commande est atteint puisque les erreurs convergent vers 0 . On peut ainsi compenser l'effet des défauts sur le système $(\tilde{x} \rightarrow 0)$ et les reproduire $(e \rightarrow 0)$ grâce au modèle interne.

\section{Tests en simulation}

\subsection{Simulation de la machine asynchrone}

Les Figures 3 et 4 illustrent la simulation en boucle ouverte des modèles élaborés pour la machine asynchrone. En premier lieu, un fonctionnement à vide avant l'application d'un couple de charge $t=1 \mathrm{sec}$. A $t=2 \mathrm{sec}$, des perturbations de $80 \%$ (par rapport aux valeurs nominales) sont introduites dans les paramètres résistifs et mécaniques de la machine.

Ensuite à $t=3 \mathrm{sec}$, on introduit un seul défaut sous forme d'un harmonique 50 $\mathrm{Hz}$, d'amplitude $8 \mathrm{~V}$ et de phase nulle (Fig. 3). Ensuite, deux défauts sont introduits, l'un dans le stator et l'autre dans le rotor, induisant trois harmoniques de fréquence 10,15 et $20 \mathrm{~Hz}$, d'amplitudes respectives $8 \mathrm{~V}, 10 \mathrm{~V}$ et $5 \mathrm{~V}$ et de phases nulles (Fig. 4). 

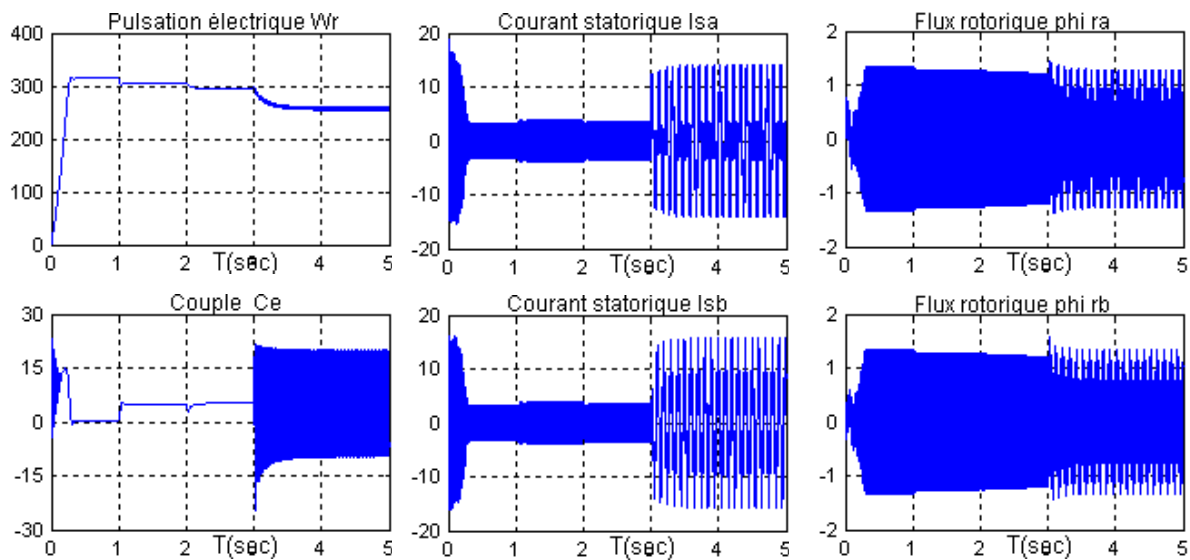

Figure 3. Effet d'un défaut.
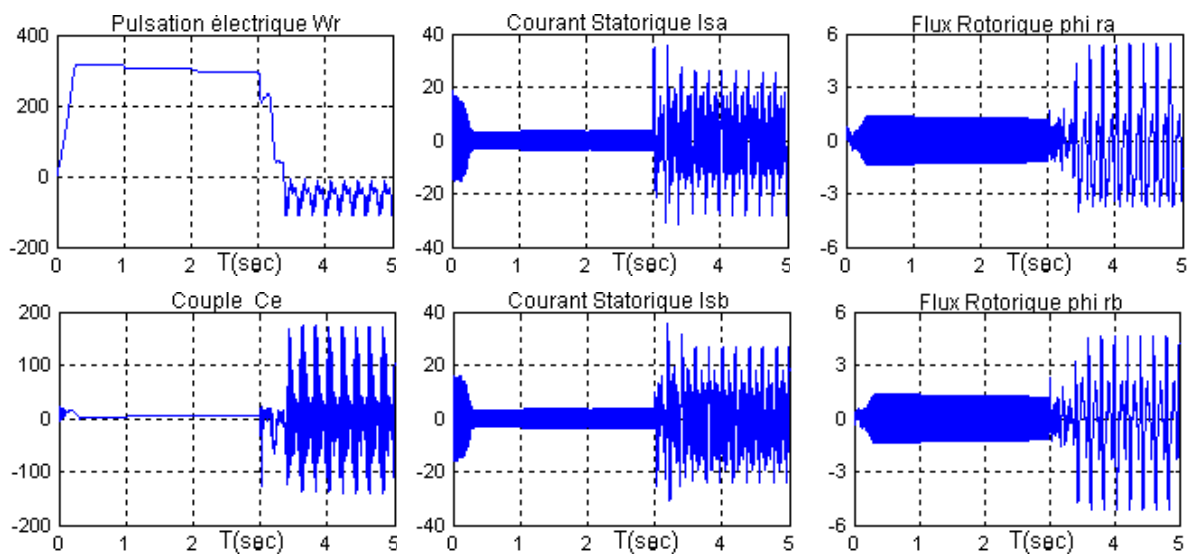

Figure 4. Effet de deux défauts.

\subsection{Test de la commande par Backstepping}

Suivant le même scénario temporel, les figures 5 à 7 illustrent les performances de la commande par Backstepping (commande nominale) proposée. Dans le cas de la figure 7 , on considère la même situation que la figure 6 (un défaut dans le stator et un autre dans le rotor) mais on augmente les gains de la commande pour augmenter la robustesse. En analysant ces résultats, on peut constater que la commande proposée est robuste par rapport aux perturbations paramétriques et au couple de charge, mais s'avère insuffisante en cas de défauts. L'augmentation de la robustesse diminue l'erreur sur la vitesse et le flux mais n'annule pas l'effet des défauts sur les courants. 

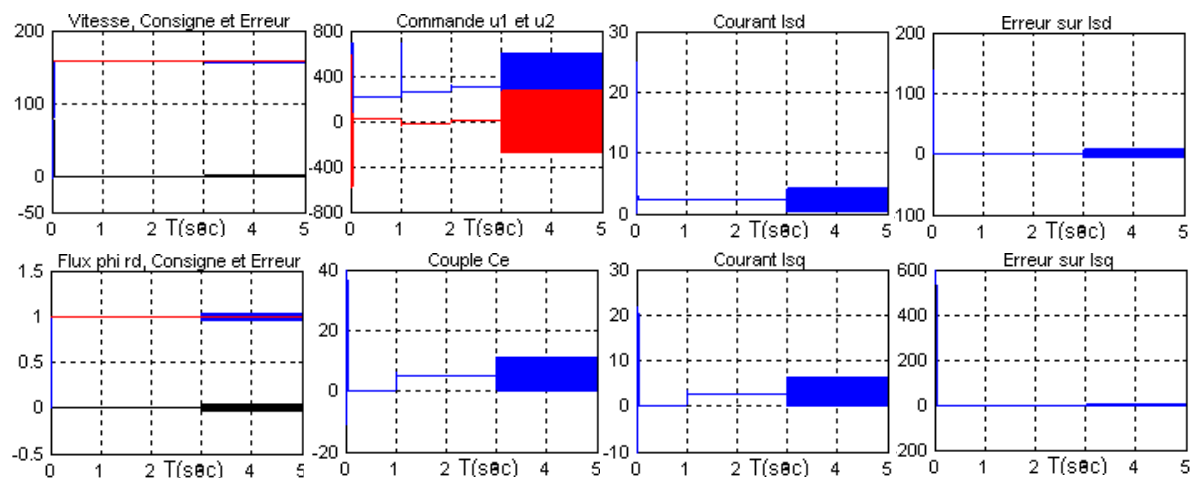

Figure 5. Commande backstepping en présence d'un seul défaut.
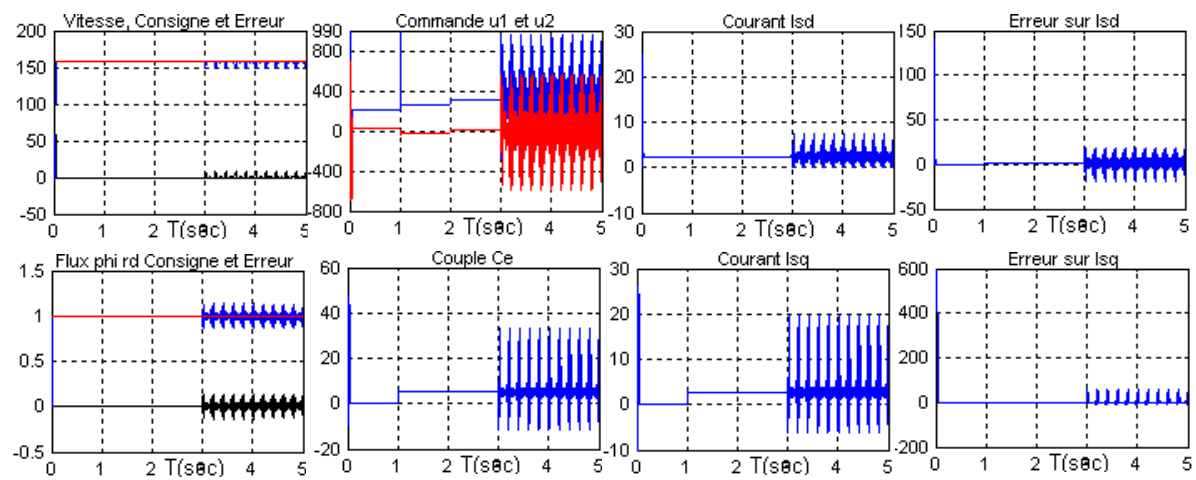

Figure 6. Commande backstepping en présence de deux défauts.
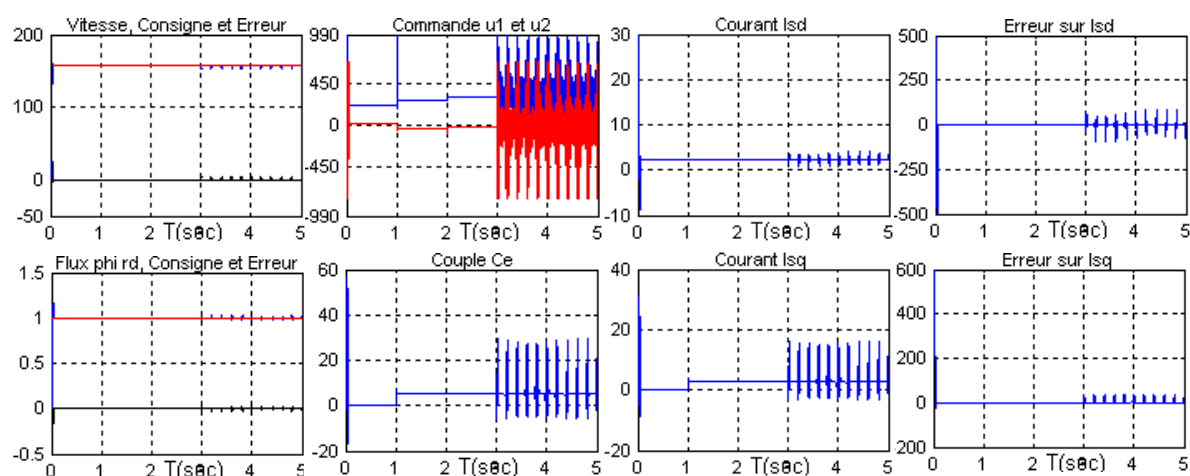

Figure 7. Présence de deux défauts avec augmentation des gains. 


\subsection{Test de la première approche FTC}

Dans ce cas, le système est simulé avec la $1^{\text {ère }}$ approche FTC en boucle fermée. Comme on peut le constater sur la figure 8 (avec le même scénario de simulation), la commande FTC calculée rejette l'effet du couple de charge, l'effet des perturbations paramétriques ainsi que l'effet des défauts. On peut noter les variations de la commande due aux termes additionnels qui suivent les variations des défauts afin de compenser leurs effets.

L'augmentation du nombre de défauts augmente les dimensions des matrices $F, S$ et $\Gamma$. Ceci pose des problèmes de conditionnement de la matrice $M$ solution de l'équation de Sylvester (Lin Y. et al., 2007) qui conduisent à des situations de non convergence.
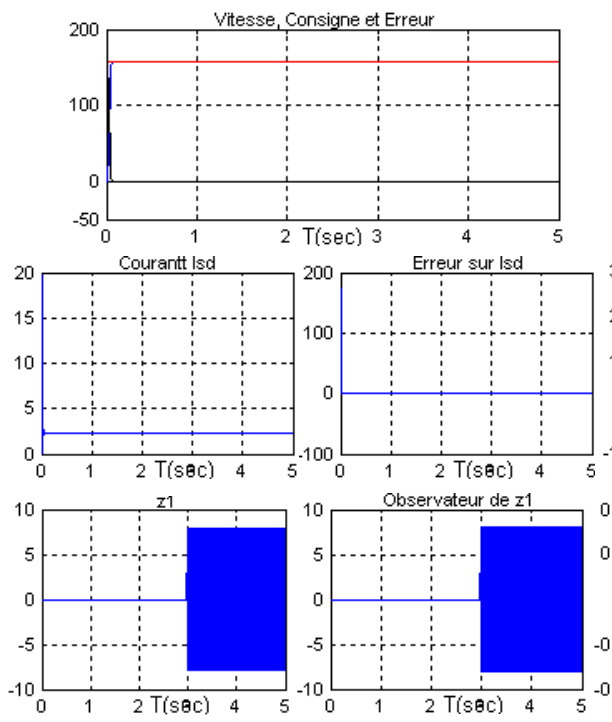
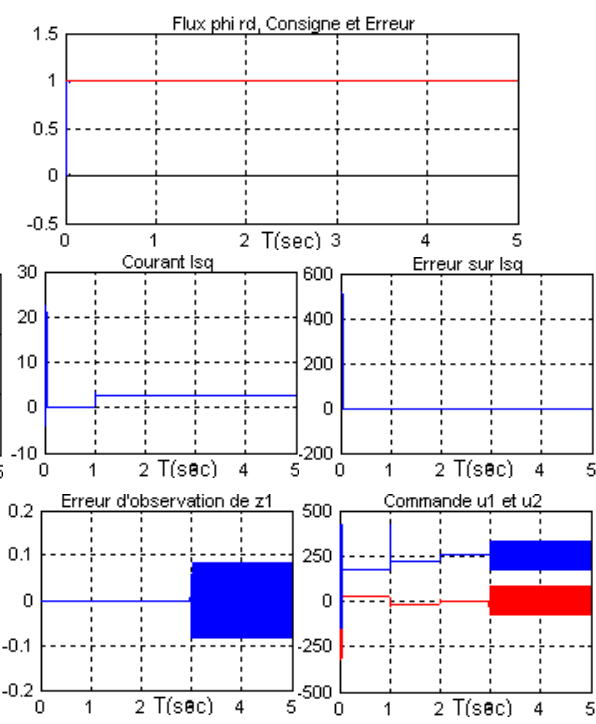

Figure 8. Commande tolérante en présence d'un seul défaut.

\subsection{Test de la seconde approche FTC}

Dans ce cas, la commande calculée rejette l'effet du couple de charge, des perturbations paramétriques ainsi que l'effet des défauts. Il n'est pas nécessaire de changer les gains de la commande pour assurer la convergence du modèle interne. Contrairement à la première approche, on a une convergence du modèle interne et on peut augmenter le nombre de défauts. Ces constats sont parfaitement illustrés par la figure 9 dans le cas d'un seul défaut (harmonique de $50 \mathrm{~Hz}$, d'amplitude $8 \mathrm{~V}$ et de phase nulle) puis par la figure 10 avec deux défauts (harmoniques de 10, 15, $20 \mathrm{~Hz}$, d'amplitudes respective $8 \mathrm{~V}, 10 \mathrm{~V}, 5 \mathrm{~V}$, et de phases nulles). 

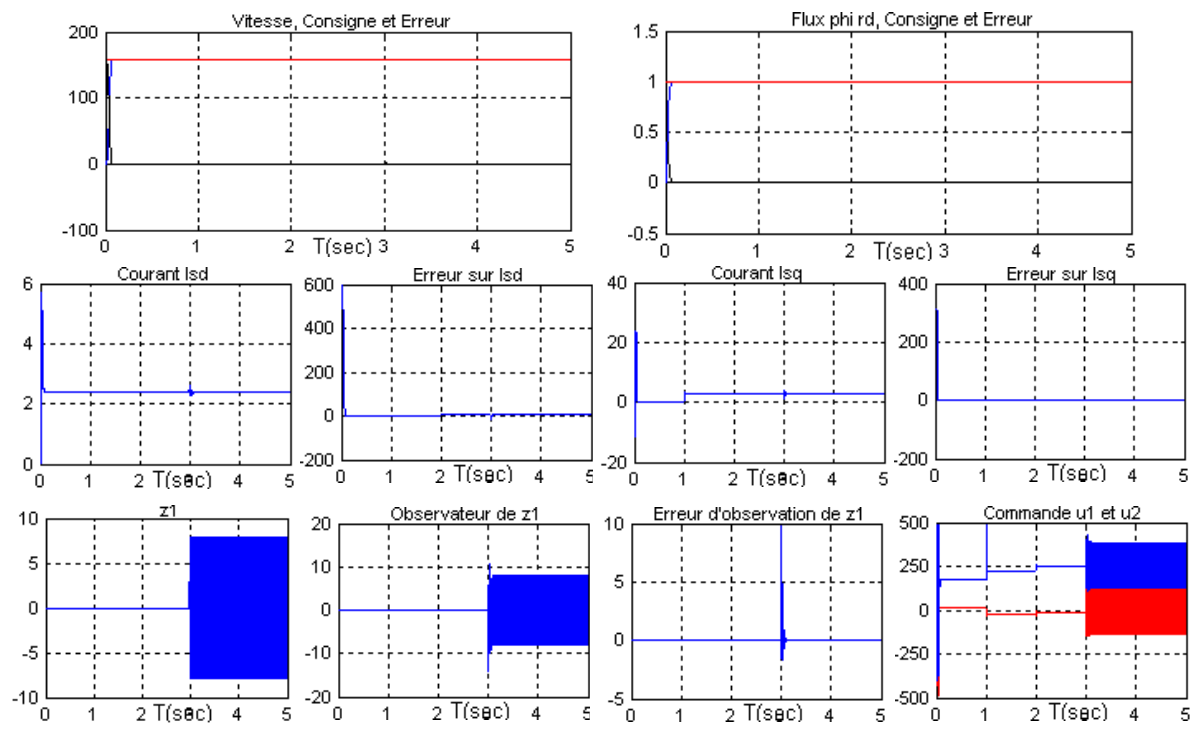

Figure 9. Commande tolérante en présence d'un seul défaut.
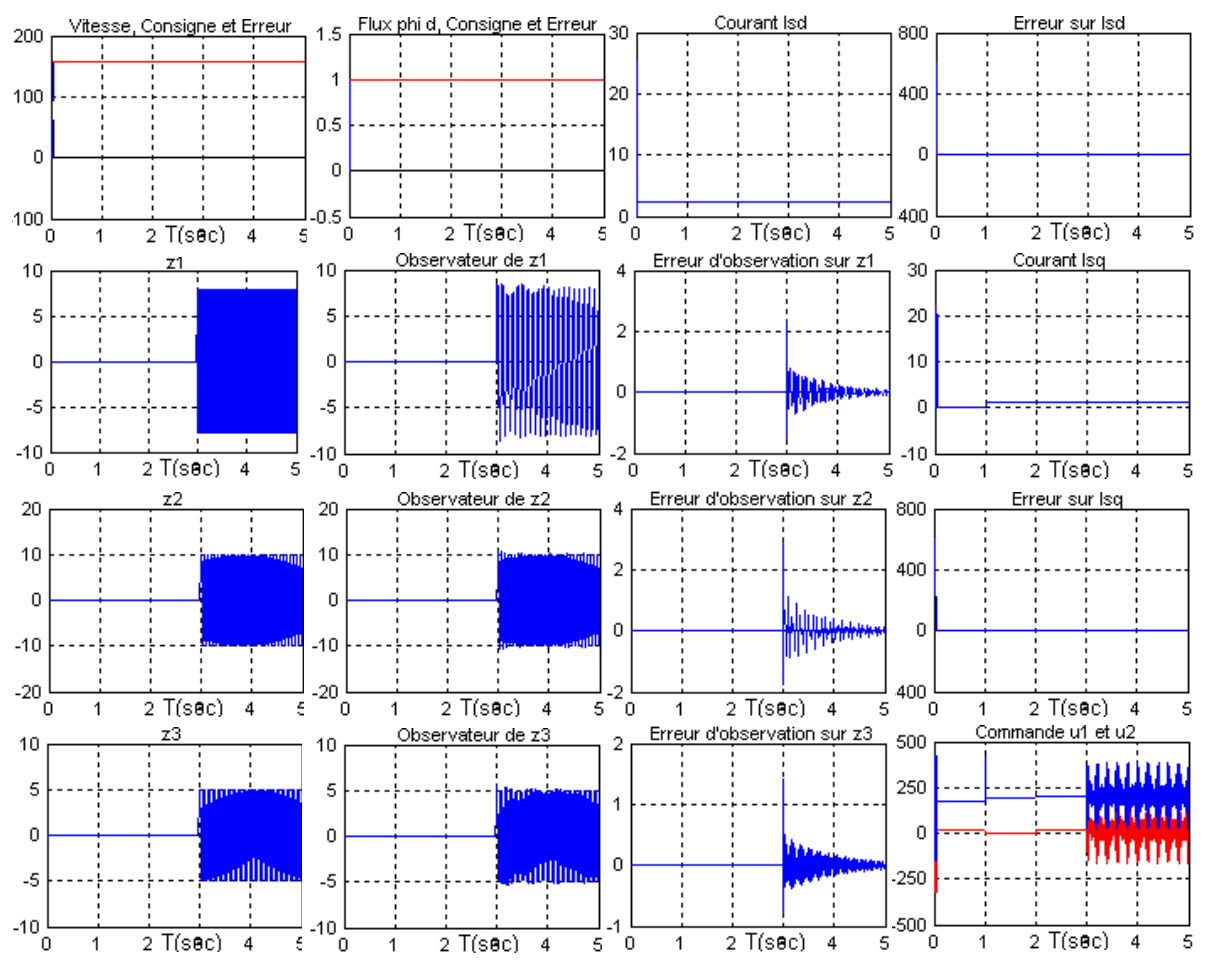

Figure 10. Commande tolérante en présence de deux défauts. 


\subsection{Comparaison des deux approches}

Le Tableau suivant établit une comparaison entre les deux approches FTC.

Tableau 1. Comparaison des deux Approches FTC.

\begin{tabular}{|c|c|}
\hline $1^{\text {ère }}$ Approche FTC & $2^{\text {ème }}$ Approche FTC \\
\hline $\begin{array}{l}\text { La résolution de l'équation de Sylvester } \\
\text { nécessite un temps de calcul important. }\end{array}$ & $\begin{array}{l}\text { L'élimination du passage par } \\
\text { l'équation de Sylvester réduit le temps } \\
\text { de calcul. }\end{array}$ \\
\hline 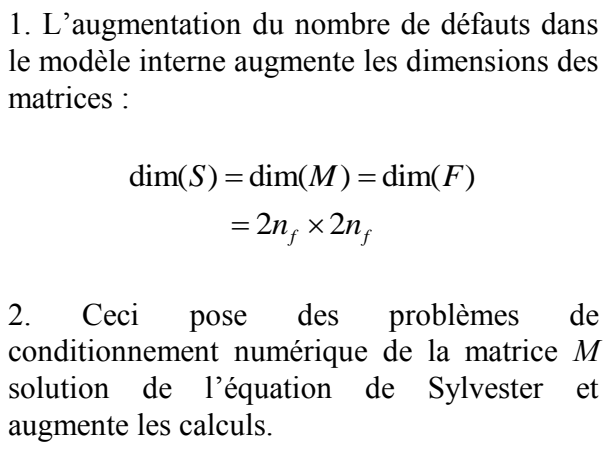 & 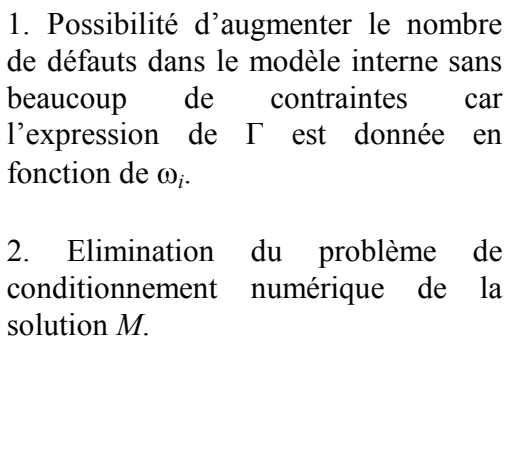 \\
\hline $\begin{array}{l}\text { Une modification éventuelle des gains de } \\
\text { réglage } k_{1} \text { et } k_{2} \text { peut être nécessaire pour la } \\
\text { convergence des calculs. }\end{array}$ & $\begin{array}{l}\text { Pas d'adaptation des gains } k_{1} \text { et } k_{2} \text { pour } \\
\text { assurer la convergence du modèle } \\
\text { interne. }\end{array}$ \\
\hline
\end{tabular}

\section{Conclusions}

Dans cet article, deux approches de commande tolérante aux défauts (FTC) ont été étudiées et appliquées à la machine asynchrone. Dans ce contexte, la phase de détection et d'isolation du défaut est décalée par rapport à la phase de reconfiguration de la commande. Celle-ci est réalisée en testant l'état d'un modèle interne qui s'active automatiquement dès l'apparition d'un défaut pour compenser son effet. Cet effet est modélisé par un signal exogène issu d'un système autonome stable appelé exosystème. Une commande additive est ainsi ajoutée à la commande par Backstepping (commande nominale) qui est robuste par rapport aux perturbations paramétriques et au couple de charge. Issue du modèle interne qui reproduit le signal défaut, la commande sert à compenser son effet.

Deux approches FTC ont été explorées : la première exploitant un modèle interne basé sur l'équation de Sylvester pose des problèmes de dimensionnement numérique de la matrice solution. Ceci a malheureusement entraîné une divergence lorsque la machine est affectée par deux défauts ou plus. La seconde approche, quant à elle, a 
éliminé ce problème de divergence par une exploitation judicieuse des matrices du problème. Les résultats de simulation sont encourageants et incitent à envisager une validation expérimentale en injectant des défauts artificiels ou en disposant d'une machine ayant de vrais défauts à signature fréquentielle.

\section{Bibliographie}

Zhang Y. et al. Bibliographical review on reconfigurable fault-tolerant control systems. Annual Reviews in Control, vol. 32, pp. 229-252, 2008.

Campos-Delgado D.U. et al. Fault-tolerant control in variable speed drives: a survey. IET Electric Power Applications, vol.2, n², pp. 121-134, March 2008.

Benbouzid M.E.H. et $a l$. What stator current processing based technique to use for induction motor rotor faults diagnosis?. IEEE Trans. Energy Conversion, vol. 18, n² ${ }^{\circ}$, pp. 238-244, June 2003.

Benbouzid M.E.H. A review of induction motors signature analysis as a medium for faults detection. IEEE Trans. Industrial Electronics, vol. 47, n5, pp. 984-993, October 2000.

Eva Wu N. Coverage in fault-tolerant control. Automatica, vol. 40, pp. 537-548, 2004.

Fekih, A. Effective fault tolerant control design for nonlinear systems: application to a class of motor control system. IET Control Theory \& Applications, vol. 2, n ${ }^{\circ}$, pp. 762-772, September 2008.

Benbouzid M.E.H. et al. Advanced fault-tolerant control of induction-motor drives for EV/HEV traction applications: From conventional to modern and intelligent control techniques. IEEE Trans. Vehicular Technology, vol. 56, n², pp. 519-528, March 2007.

Jeong Y.S. et al. Fault detection and fault-tolerant control of interior permanent-magnet motor drive system for electric vehicle. IEEE Trans. Industry Applications, vol. 41, ${ }^{\circ} 1$, pp. 46-51, January-February 2005.

Bonivento C. et al. Implicit fault-tolerant control: Application to induction motors. Automatica, vol. 40, pp. 355-371, 2004.

Tadjine M. et al. Robust backstepping vector control for the doubly fed induction motor. IET Control Theory \& Applications, vol. 1, n4, pp. 861-868, July 2007.

Ebrahim A. et al. Adaptive backstepping control of an induction motor under time-varying load torque and rotor resistance uncertainty. In Proceedings of the IEEE SSST'06, Cookville, (USA), pp. 512-518, March 2006.

Tan H. et al. Adaptive backstepping control of induction motor with uncertainties. In Proceedings of the IEEE ACC'99, San Diago (USA), vol. 1, pp. 1-5, June 1999.

Krause P.C. et al. Analysis of Electric Machinery and Drive Systems. John Wiley \& Sons, 2002.

Leonhard W. Control of Electrical Drives. Springer-Verlag, 1996. 
Muenchhof M. et al. Fault-tolerant actuators and drives-Structures, fault detection principles and applications. Annual Reviews in Control, October 2009.

O'Leary D.P. Fast solvers and Sylvester equations: both sides now. Computing in Science \& Engineering, vol. 7, nº, pp. 74-80, November-December 2005.

Lin Y. et al. Condition numbers of the generalized Sylvester equation. IEEE Trans. Automatic Control, vol. 52, ${ }^{\circ} 12$, pp. 2380-2385, December 2007. 